\title{
The solar eruption of 13 May 2005: EISCAT and MERLIN observations of a coronal radio burst
}

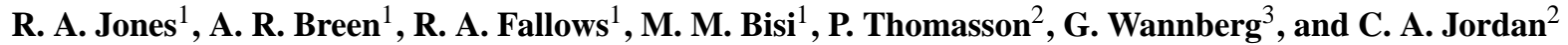 \\ ${ }^{1}$ Institute of Mathematical and Physical Sciences, University of Wales, Aberystwyth, UK \\ ${ }^{2}$ Jodrell Bank Observatory, Manchester University, UK \\ ${ }^{3}$ EISCAT Scientific Association, Kiruna, Sweden
}

Received: 13 December 2005 - Revised: 10 February 2006 - Accepted: 13 February 2006 - Published: 20 September 2006

Part of Special Issue "Twelfth EISCAT International Workshop"

\begin{abstract}
We report results from EISCAT and MERLIN observations of radio scintillation during a solar eruptive event in May 2005. Anomalous increases in signal strength detected at sites more than $2000 \mathrm{~km}$ apart are shown to arise from the detection of a strong coronal radio burst in the distant off-axis response of the MERLIN and EISCAT antennas. These observations show that EISCAT is capable of detecting the signatures of explosive events in the solar atmosphere with a high degree of time resolution. We further suggest that the highly time-structured variation in signal strength caused by distant off-axis detection of a powerful coronal radio signal could provide an explanation for previously unexplained anomalies in EISCAT IPS observations, as well as being a potential source of errors in active observations using radar codes with a completion time longer than the time-variation of the coronal signal.
\end{abstract}

Keywords. Solar physics, astrophysics, and astronomy (Radio emissions; Flares and mass ejections) - Radio science (Instruments and techniques)

\section{Introduction}

Measurements of interplanetary scintillation (IPS) have been used to study the solar wind for over forty years (e.g. Hewish et al., 1964; Armstrong and Coles, 1972; Kojima and Kakinuma, 1990). With its wide antenna separations, lownoise receivers and accurate timing control, EISCAT is wellsuited to IPS observations (Bourgois et al., 1985) and regular programmes of measurements have taken place since 1989 (Coles et al., 1991). The MERLIN network of radio telescopes has also been used for IPS studies (Rickett, 1992; Breen et al., 2000) and from 2002 the two systems have been

Correspondence to: A. R. Breen

(azb@aber.ac.uk) operated together as a single extremely long-baseline system for a limited number of observations each year (Breen et al., 2006).

IPS arises when a compact astronomical radio source is observed close to the Sun in the sky. Differences in the degree of refraction introduced by turbulent-scale irregularities give rise to phase changes in the incident wave - these are converted to amplitude variation as the signal continues to propagate, and if this pattern is sampled with two radio telescopes then the result will be two time-series of signal power, with the signal varying on timescales of $0.1-10 \mathrm{~s}$. The accuracy to which IPS can estimate solar wind speed improves as the separation of the antennas projected into the plane of the sky increases (Rao et al., 1995; Coles, 1996). The programme of extremely long baseline IPS experiments using EISCAT and MERLIN takes advantage of this improved sensitivity to study the fine structure of solar wind velocity distribution (Bisi et al., 2006; Breen et al., 2006).

IPS observations require accurate measurements of signal power with a time resolution sufficient to determine not only the spectrum of scintillation, but the background level at higher frequencies. EISCAT samples the detected signal at a rate of $10^{4} \mathrm{~Hz}$ which is subsequently averaged to give a low-noise time series of intensity measurements at 0.01 -s intervals, while MERLIN samples at $100 \mathrm{~Hz}$. EISCAT IPS observations have occasionally detected anomalous bursts of enhanced radio signal intensity with little or no timelag between the variations at different sites. Until recently the cause of these events was assumed to be solar, but this assumption had not been tested. In this paper we discuss one such event which was recorded simultaneously at EISCAT and MERLIN. The availability of data at two frequencies from the very widely separated antennas of EISCAT and MERLIN, together with supporting information from the GOES, SoHO and TRACE spacecraft and from the PHOENIX-2 radio spectrometer made it possible to

Published by Copernicus GmbH on behalf of the European Geosciences Union. 


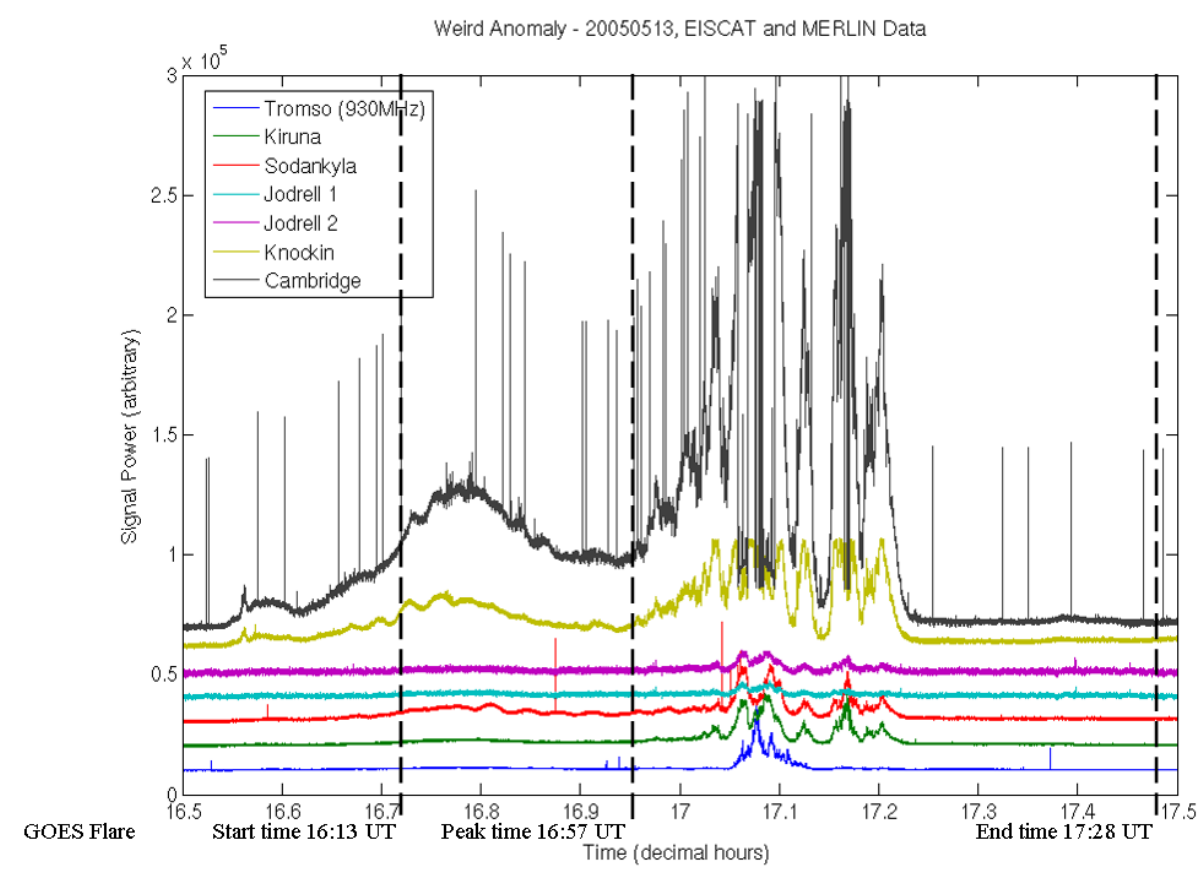

Fig. 1. Observations from EISCAT and MERLIN of the anomalous radio signal strength enhancement of 13 May 2005. The Jodrell Bank (Mk.1A), Cambridge and Knockin telescopes of MERLIN and the Kiruna and Sodankylä telescopes of EISCAT were operating at a central frequency of $1.4 \mathrm{GHz}$. The Troms $\varnothing$ telescope of EISCAT was operating at a central frequency of $0.93 \mathrm{GHz}$.

characterise this event as a coronal radio burst, detected in the distant side-response of the antenna beams. A coronal mass ejection launched by this event was detected by EISCAT and MERLIN during IPS observations the following day and went on to pass the ACE spacecraft at the L1 Lagrange point at 02:00 UT on 15 May 2005. Analysis of this event is in progress and it will be described in a forthcoming paper.

\section{Observations on 13 May 2005}

Shortly after 16:30 UT on 13 May 2005 during IPS observations of the strong radio source $0319+415$ (3C84) the telescopes of EISCAT and MERLIN observed significant and highly time-variant enhancements in signal strength (Fig. 1). This enhancement lasted until shortly after 17:10 UT on 13 May 2005. X-ray data from the GOES spacecraft and extreme ultraviolet images from the EIT instrument on SoHO (Fig. 2) showed that a significant solar eruption had taken place near disc centre, beginning shortly after 16:30 UT. At the same time the PHOENIX-2 radio spectrometer (Messmer et al., 1999) recorded a strong radio burst from the solar corona (Fig. 3), with emission moving to lower frequencies with time. Taken together, these observations immediately suggested that the enhancement in signal strength detected by EISCAT and MERLIN were associated with the solar eruption and could, perhaps, represent a detection of the coronal radio emission. There did, however, appear to be some problems with this interpretation.

On 13 May 2005 the point of closest approach of the raypaths from the EISCAT and MERLIN telescopes to 3C84 lay 84 solar radii $\left(R_{S}\right)$ off the north-east limb of the Sun - over $20^{\circ}$ away from the Sun in the sky. The main beam of the Kiruna and Sodankylä EISCAT UHF antennas operating at $1.4 \mathrm{GHz}$ is $0.44^{\circ}$ wide, with first sidelobes some $17 \mathrm{~dB}$ down on the main beam (Wannberg et al., 2002). This appeared to make it unlikely that a source so far off-axis could be detected. Furthermore, early EISCAT observations of IPS at 10-12 $R_{s}$ from Sun centre showed a very clear "saw-tooth" pattern in signal strength, caused by the limb of the Sun drifting in and out of the beam sidelobes as the antenna moved every $2.56 \mathrm{~s}$ (Breen et al., 1996). This led us to expect that if the source had been detected in a side-lobe of the antenna beam then it would have shown a $2.56 \mathrm{~s}$ periodicity in EISCAT observations but not in data from MERLIN, where the antennas track continuously. This was not the case, and it made it considerably harder to explain the observations. Further difficulties arose from the difference in strength and in time-variation of the signal enhancement recorded at the different sites. These issues are discussed in the next section.

\section{Discussion}

The structure and strength of the enhancement event seen in radio data from EISCAT and MERLIN differed from 


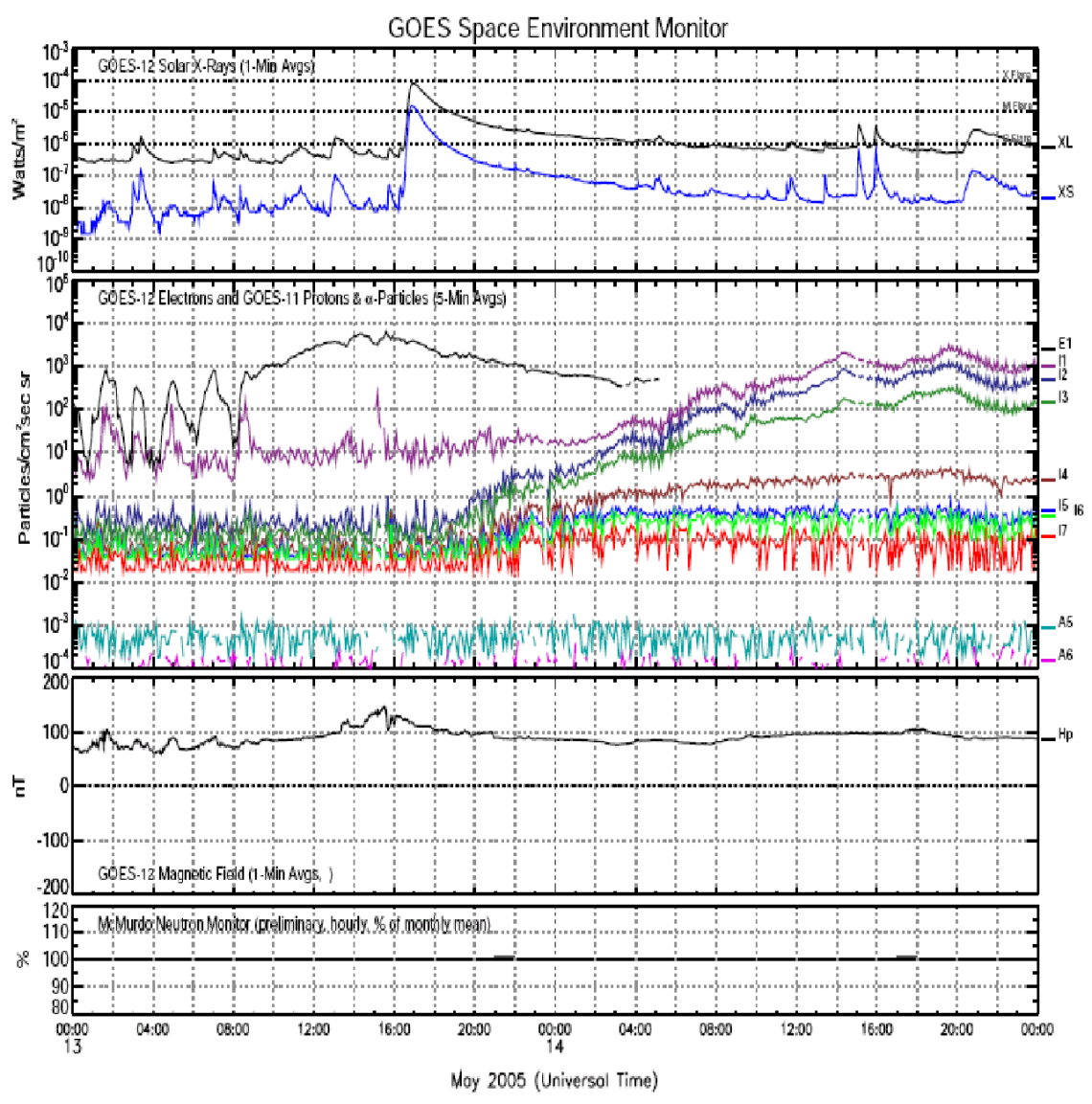

Fig. 2. GOES X-ray flux (top panel) on 13 and 14 May 2005. The onset of the flare shortly after 16:30 UT on 13 May 2005 can be clearly seen.

telescope to telescope. In particular, the time-series of signal power from Cambridge showed a high degree of fine structure between shortly after 17:00 UT and 17:15 UT which was not present in data from the other telescopes. The strength of the enhancement was also greatest in the data from these two telescopes, with the largest increase in signal strength seen at Cambridge. The degree of enhancement was considerably weaker in the EISCAT $1.4 \mathrm{GHz}$ measurements from Kiruna and Sodankylä, while the $0.93 \mathrm{GHz}$ measurements from Troms $\varnothing$ showed a distinctly different time variation. The enhancement was weakest in the data from the $76 \mathrm{~m}$ Jodrell Bank Mk.1A telescope, though the time variation was very similar to that seen in the EISCAT $1.4 \mathrm{GHz}$ measurements.

While initially puzzling, these variations proved possible to explain in terms of the known characteristics of the two systems:

- The 76m Mk.1A telescope at Jodrell Bank has a considerably narrower beam then the $32 \mathrm{~m}$ EISCAT antennas or the $25 \mathrm{~m}$ and $32 \mathrm{~m}$ telescopes at the other MERLIN sites. The weaker response in the Mk.1A signal was an important indication that the radio enhancement did not lie in the centre of the telescope beams;

- the rapid time-variation of the signal seen in the Cambridge data is consistent with the known characteristics of the MERLIN signal processing hardware, which instead of saturating "rolls over" and resumes counting from zero. This rapid time variation - which was not seen in the weaker signal strength enhancement seen at the other elescopes - is therefore an artifact introduced when the enhancement raised signal levels out of acceptable limits for MERLIN;

- the weaker response seen in EISCAT $1.4 \mathrm{GHz}$ data and particularly in Kiruna data - is consistent with the results of other IPS observations in 2005, in which the signal strength at Kiruna was lower than observed in previous years;

- the difference in the time-variation of the strength of enhancement seen at Troms $\varnothing$ at $0.93 \mathrm{GHz}$ and in the other sites at $1.4 \mathrm{GHz}$ is easily understood if the source of emission was itself varying in frequency during the event. 


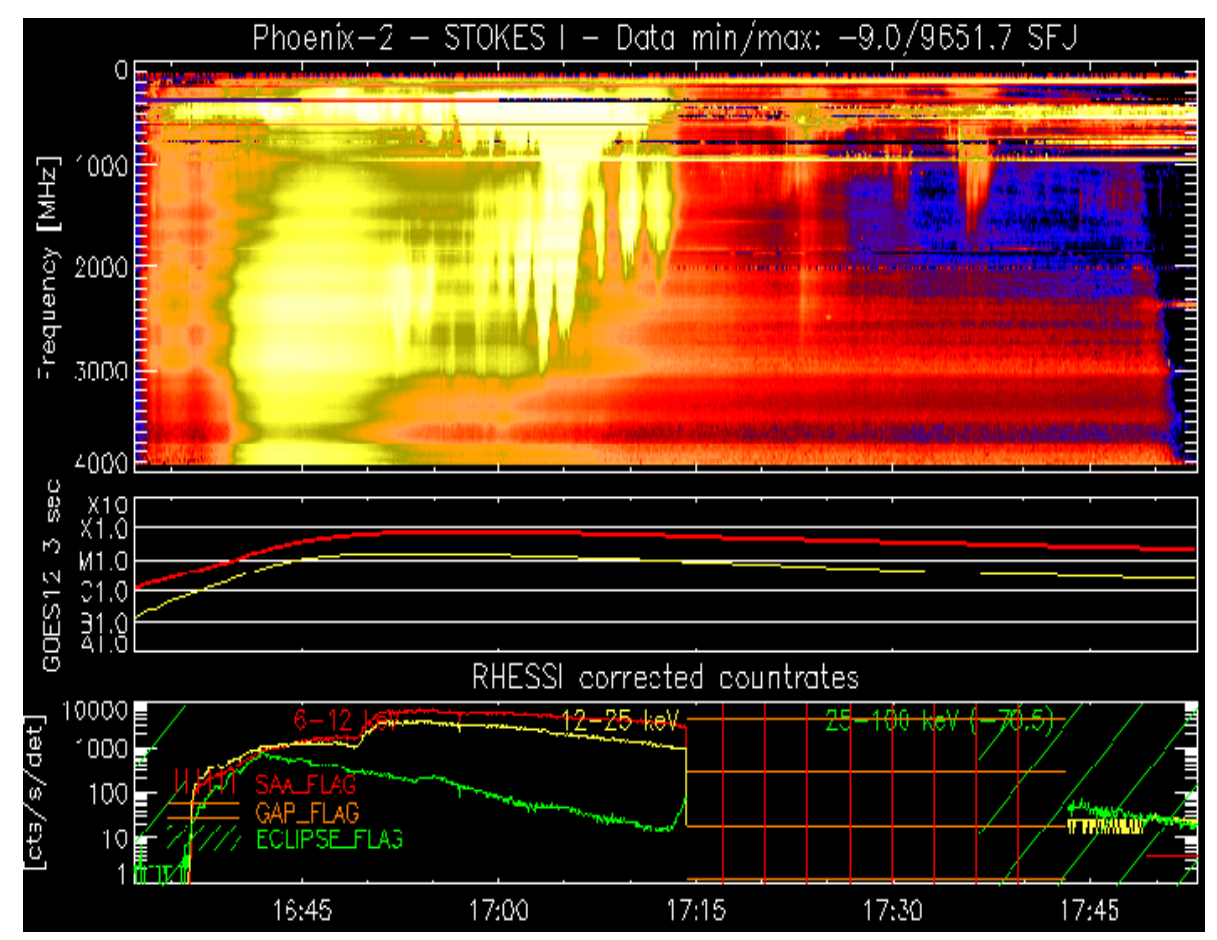

Fig. 3. PHOENIX-2 radio spectrogram from 16:30 UT onwards on 13 May 2005. The onset of enhanced emission at lower frequencies from 16:35 UT, leading to a strong burst from 16:45 UT can be clearly seen. The evolution from high-frequency emission to lower frequencies is characteristic of coronal radio bursts, as the blast wave from the explosive event rises higher into the corona.

Table 1. Results of cross-correlating time series of signal intensities recorded at the EISCAT and MERLIN sites against PHOENIX-2 observations The data were averaged over $30 \mathrm{~s}$ (shown in bold type), $10 \mathrm{~s}$ (shown in normal type) and $0.1 \mathrm{~s}$ (shown in italic type). The $30 \mathrm{~s}$ and $10 \mathrm{~s}$ averages were used to eliminate the short-period time-variation due to interplanetary scintillation from the EISCAT and MERLIN data, the 0.1-s averaging was used to determine whether any time-lag between variations in EISCAT/MERLIN and PHOENIX data was present.

\begin{tabular}{|c|c|c|c|}
\hline Site 1 & Site 2 & $\begin{array}{l}\text { Maximum cross- } \\
\text { correlation \% Data } \\
\text { averaged over: } \\
\text { 30 s, } 10 \mathrm{~s}, 0.1 \mathrm{~s}\end{array}$ & $\begin{array}{l}\text { Time-lag for maximum } \\
\text { cross-correlation (s) }\end{array}$ \\
\hline \multirow[t]{6}{*}{ PHOENIX-2 1.4 GHz } & Kiruna & $\mathbf{6 0 . 1}, 59.7,59.2$ & $\mathbf{0}, 0,0.0$ \\
\hline & Sodankylä & $66.2,64.6,64.1$ & $\mathbf{0}, 0,0.0$ \\
\hline & Jodrell Bank channel 1 & $66.3,64.8,58.8$ & $\mathbf{0}, 0,0.0$ \\
\hline & Jodrell Bank channel 2 & 62.8, $62.1,58.6$ & $\mathbf{0}, 0,0.0$ \\
\hline & Cambridge & 77.0, $76.4,71.3$ & $\mathbf{0}, 0,0.0$ \\
\hline & Knockin & 73.9, 71.7, 70.4 & $\mathbf{0}, 0,0.0$ \\
\hline PHOENIX-2 $0.915 \mathrm{GHz}$ & Troms $\varnothing$ & 89.6, $91.3,89.8$ & $\mathbf{0}, 0,0.0$ \\
\hline PHOENIX-2 $0.985 \mathrm{GHz}$ & & $\mathbf{5 9 . 7}, 60.0,60.5$ & $\mathbf{0}, 0,0.0$ \\
\hline
\end{tabular}

Once the differences between the observing systems were taken into account, the results provided strong evidence that all the telescopes receiving at $1.4 \mathrm{GHz}$ were observing an event with the same characteristics, that the source of emission did not lie in the centre of the beam and that there was an intrinsic difference between the characteristics of emission at 0.93 and $1.4 \mathrm{GHz}$. These characteristics seemed to be consistent with an observation of a coronal radio burst which was co-temporal with the flare, but we were initially uncertain of this interpretation because the observation was 
so far off-Sun and because no signatures of the tracking interval (such as we would have expected if strong emission was being picked up in a discrete side-lobe) were apparent in the EISCAT data. W. Coles and W. Erickson independently suggested to us that we might be detecting strong emission from the corona in the relatively flat, far off-axis response of the telescopes (private communications, 2005), an interpretation which was supported by detailed consideration of the EISCAT UHF beam characteristics at $1.4 \mathrm{GHz}$, which showed that by $20^{\circ}$ off-axis the response was near-constant at about $10^{-5}$ of the gain at beam centre. With this established, we could confidently associate the transient enhancement in signal strength detected by EISCAT and MERLIN on 13 May 2005 with the coronal radio burst detected by PHOENIX-2.

The IPS experiment which provided that data discussed in this paper was primarily designed to record fluctuations scintillations - in signal strength taking place on timescales of $\sim 0.1$ to $10 \mathrm{~s}$. The time-structure of the radio burst, however, varies more slowly than this, so before comparing the variation in signal power seen by EISCAT and MERLIN with the PHOENIX-2 record varying degrees of smoothing were introduced by taking running averages over periods of $30 \mathrm{~s}$, 10 s (to smooth out the short-period fluctuations in EISCAT and MERLIN data introduced by interplanetary scintillation) and $0.1 \mathrm{~s}$ (to determine whether there was any time-lag between variations seen by the two systems). When the $10 \mathrm{~s}-$ averaged time-series of signal intensities from MERLIN and EISCAT (Kiruna and Sodankylä) data were cross-correlated against the PHOENIX-2 time-series at $1.415 \mathrm{MHz}$ strong correlations at zero lag were revealed, ranging from $76.4 \%$ between PHOENIX-2 and Cambridge data and $59.7 \%$ between PHOENIX-2 and Kiruna (Table 1). The agreement between the PHOENIX-2 records at $0.915 \mathrm{GHz}$ and EISCAT Troms $\varnothing$ at $0.928 \mathrm{GHz}$ was even better, showing a correlation of over $91 \%$. The degree of correlation between the EISCAT record and the PHOENIX-2 $0.985 \mathrm{GHz}$ time-series was. There was no evidence for any time-lag for maximum correlation, even when the averaging period was reduced to $0.1 \mathrm{~s}$ (Table 1).

Figure 4 compares the intensity of radio emission detected at EISCAT and MERLIN sites with that measured at 0.93 and $1.4 \mathrm{GHz}$ by PHOENIX-2. The agreement is most impressive, strongly indicating that the instruments were observing the same event. An especially interesting point is that EISCAT and MERLIN provide better information on the peak of the radio burst - the PHOENIX instrument reaches saturation, but EISCAT and MERLIN (with the exception of Cambridge) remain within signal intensity limits. This result clearly demonstrates that EISCAT (and MERLIN) can provide valuable information on the evolution of coronal radio busts - and thus on solar explosive events.

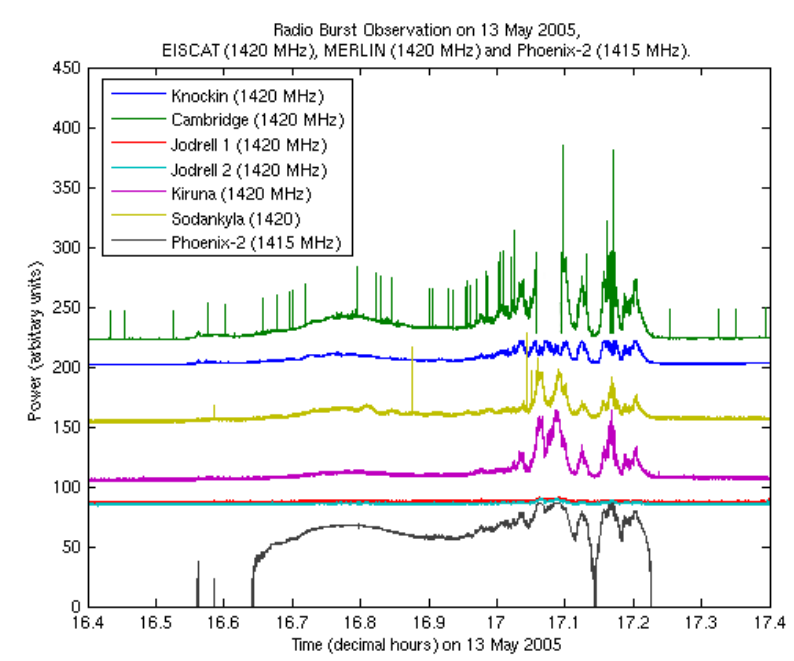

(a)

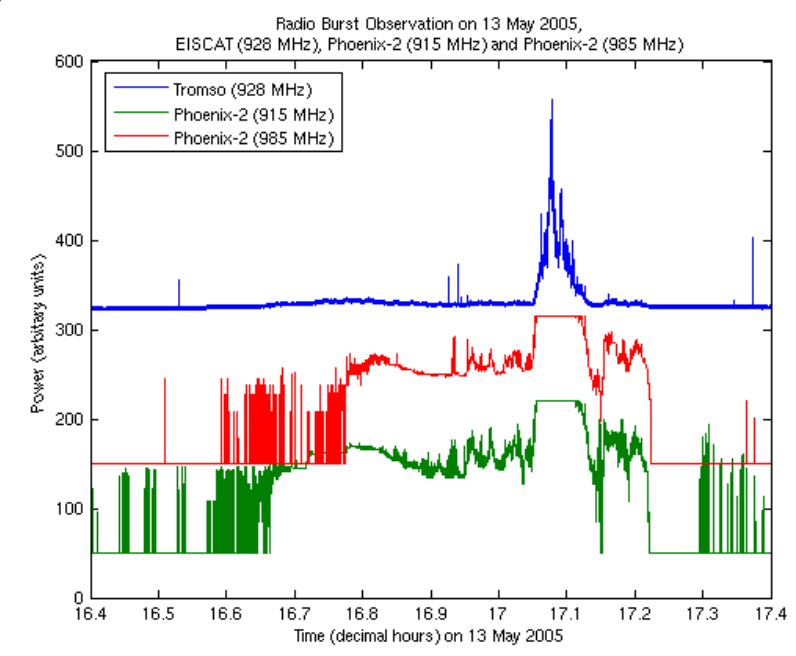

(b)

Fig. 4. (a) Time-series of radio intensity recorded at the Kiruna and Sodankylä EISCAT sites, at the Jodrell Bank, Knockin and Cambridge MERLIN sites and by the PHOENIX-2 instrument at ETH Zurich, all at $1.4 \mathrm{GHz}$. (b) Time-series of radio intensity recorded at EISCAT Troms $\varnothing$ at $0.928 \mathrm{GHz}$ and by the PHOENIX-2 instrument at ETH Zurich at 0.915 and $0.985 \mathrm{GHz}$.

\section{Conclusions}

The results presented in this paper are clear evidence that the anomalous enhancement in EISCAT and MERLIN signal levels detected on 13 May 2005 were due to the detection of a coronal radio burst in the distant side response of the antenna beams. In particular, the data from Sodankylä, Knockin and Jodrell Bank show very good agreement with the time-series of signal intensity at $1.415 \mathrm{GHz}$ recorded by the PHOENIX-2 radiospectrograph - and provide better information on the 
time-variation of most intense period of the radio burst. The Troms $\varnothing$ record at $0.928 \mathrm{GHz}$ provides a valuable intermediate frequency between two of the PHOENIX-2 bands and again - provides exceptionally good coverage of the period of maximum emission during the burst. We consider that these results show that EISCAT is capable of making a valuable contribution to studies of the upper atmosphere of the Sun, as well as that of the Earth.

Anomalous periods of enhanced signal strength have been detected in EISCAT IPS observations in the past, and although studies are still underway we now believe that these too are likely to be associated with solar eruptive events.

Finally, we would like to suggest that the detection of a coronal radio burst in the distant off-axis response of the EISCAT antennas may have implications for ionospheric studies using the radar. The current tau-2 16-bit alternating codes used for F-region observations with the EISCAT UHF system have a "stationarity" time - for which the radar target is assumed to remain invariant - of approximately $0.5 \mathrm{~s}$. The variation in intensity of the radio burst, as detected at EISCAT, showed structure on similar timescales. The increase in signal intensity recorded by PHOENIX-2 at $0.985 \mathrm{GHz}$ was over $11.6 \mathrm{Jy}\left(11.6 \times 10^{-26} \mathrm{~W} \mathrm{~m}^{-2} \mathrm{~Hz}^{-1}\right)$, so the excess power received by one of the $32-\mathrm{m}$ antennas of EISCAT across a receiver bandwidth of $100 \mathrm{kHz}$ would be of the order of a few $10^{-17} \mathrm{~W}$. Typical IS returns during daytime are of the order of $10^{-12} \mathrm{~W}$, so under normal daytime ionospheric conditions the radio burst discussed in this paper would be unlikely to cause problems for active EISCAT observations. However, stronger radio bursts detected under conditions of low ionospheric electron density could potentially pose problems for active observations if more complex codes with longer stationarity times are introduced, particularly for observations of altitudes above the F2 peak made when the Sun lies close in the sky to the ray-path of the radar beam.

Acknowledgements. We would like to thank the Director and staff of EISCAT and Jodrell Bank for the their invaluable assistance in carrying this programme through and for the data used in this study. EISCAT is supported by the scientific research councils of Finland, France, Germany, Japan, Norway, Sweden and the UK. MERLIN is a UK National facility operated by the University of Manchester on behalf of PPARC.

PHOENIX-2 data were made available by the Radio Astronomy and Plasma Physics group of the Swiss Federal Institute of Technology, Zurich (ETZ) and we would like to acknowledge this important contribution to this paper.

We would like to extend particular thanks to W. A. Coles, W. Erickson, B. Rickett, I. W. McCrae and V. Howells for making their expertise and advice available to us. R. A. Fallows, M. M. Bisi and R. A. Jones were supported by PPARC during the period when this work was carried out.

Topical Editor R. J. Forsyth thanks W. A. Coles and another referee for their help in evaluating this paper.

\section{References}

Armstrong, J. A. and Coles, W. A.: Analysis of three-station interplanetary scintillation data, J. Geophys. Res., 77, 4602-4610, 1972.

Bisi, M. M., Breen, A. R., Habbal, S. R., Fallows, R. A., and Jones, R. A.: "Large-scale structure of the fast solar wind", J. Geophys. Res, submitted, 2006.

Bourgois, G., Coles, W. A., Daign, G., Silen, J., Turenen, T., and Williams, P. J. S.: Measurements of solar wind velocity using EISCAT, Astron. Astrophys., 144, 452-462, 1985.

Breen, A. R., Tappin, S. J., Jordan, C. A., Thomasson, P., Moran, P. J., Fallows, R. A., Canals, A. and Williams, P. J. S.: Simultaneous interplanetary scintillation and optical measurements of the acceleration of the slow solar wind, Ann. Geophys., 18, 9951002, 2000.

Breen, A. R., Fallows, R. A., Bisi, M. M., Thomasson, P., Jordan, C. A., Wannberg, G., and Jones, R. A.: Extremely long-baseline interplanetary scintillation measurements of solar wind velocity, J. Geophys. Res., in press, 2006.

Coles, W. A., Esser, R., Løvhaug, U.-P., and Markkanen, J.: Comparison of solar wind velocity measurements with a theoretical acceleration model, J. Geophys. Res., 96, 13 849-13 859, 1991.

Coles, W. A.: A bimodal model of the solar wind, Astrophys. Space Sci., 243(1), 87-96, 1996.

Hewish, A., Scott, P. F., and Willis, D.: Interplanetary scintillation of small-diameter radio sources, Nature, 203, 1214-1217, 1964.

Kojima, M. and Kakinuma, T.: Solar-cycle dependence of global distribution of solar wind speed, Space Sci. Rev., 53, 173-222, 1990.

Messmer, P., Benz, A. O., and Monstein, C.: PHOENIX-2: A new broadband spectrometer for decimetric and microwave radio bursts. First results, Sol. Phys., 187(2), 335-345, 1999.

Rao, A. P., Ananthakrishnan, S., Balasubramanian, V., and Oberoi, D.: Very long baseline IPS observations of the solar wind speed in the fast polar streams, Proc. of the 8th international "Solar Wind Eight Conference”, Danapoint CA, AIP Conf. Proc. 382, $511,1995$.

Rickett, B.: IPS observations of the solar wind velocity and microscale density irregularities in the inner solar wind, in: Solar Wind 7, Proceedings of the 3rd COSPAR Colloquium held in Goslar, Germany, 16-20 September 1991, edited by: Marsch, E. and Schwenn, R., COSPAR colloquium series, 3, 255-258, 1992.

Wannberg, G., Vanhainen, L.-G., Westman, A., Breen, A. R., and Williams, P. J. S.: The new $1420 \mathrm{MHz}$ dual-polarisation interplanetary scintillation (IPS) facility at EISCAT, Proc. Union of Radio Scientists (URSI) 2002, 1144-1147, 2002. 